\title{
Redefining the Significance of Aneuploidy in the Prognostic Assessment of Colorectal Cancer
}

\author{
Rosa-Ana Risques, Victor Moreno, Eugenio Marcuello, Jordi Petriz, \\ Jose Antonio Cancelas, Francesc J. Sancho, Àngels Torregrosa, \\ Gabriel Capellà, and Miguel A. Peinado
}

Institut de Recerca Oncològica (RAR, JP, JAC, ÀT, MAP) and Institut Català d'Oncologia (VM), Hospital Duran i Reynals, L'Hospitalet, and Servei d'Oncologia Médica (EM, GC), Servei d'Anatomia Patològica (FJS), and Laboratori d'Investigació Gastrointestinal (GC), Hospital de la Santa Creu i Sant Pau, Barcelona, Spain

SUMMARY: The aberrant content of DNA, or aneuploidy, is a hallmark of tumor cells and may be associated with malignant potential. Based on the hypothesis that aneuploidy, as a form of genetic instability, results in an increased capability to generate cell heterogeneity, we investigated whether a comprehensive assessment of aneuploidy extent and degree might be a reliable indicator of tumor aggressiveness. DNA content was determined by flow cytometry in the infiltrating front of 131 paraffinembedded primary colorectal carcinomas collected in a prospective design. Enrichment of tumor cells by sample microdissection resulted in neoplastic cell contents above $75 \%$. An estimate of aneuploidy, the aneuploidy index (Al), was calculated as the tumor DNA content adjusted by the percentage of diploid and aneuploid cells in G0/G1. Thirty-nine tumors were diploid, 90 hyperdiploid, and 2 hypodiploid. The mean Al in aneuploid tumors was $1.20 \pm 0.17$ and correlated with Dukes' stage and metastasis $(p<0.05)$. A high Al (receiver operating characteristic curve cutoff value greater than 1.14) predicted a poorer outcome in univariate $(p=0.004)$ and multivariate $(p=0.01)$ analyses. Based on these results, we postulate that aneuploidy is the molecular engine of progression in a subset of colorectal cancers, in which the Al seems to be a sensible and independent gauge of malignant potential. The Al determination may have prognostic application in colorectal cancer, especially in low-grade tumors, which might benefit from coadjuvant therapies. (Lab Invest 2001, 81:307-315).

$$
T
$$
he extents of tumor infiltration of the bowel wall and lymph node metastases, both included in the Dukes' stage and tumor node metastasis (TNM) classification systems, are the most important prognostic factors in colorectal cancer (Crissman et al, 1989; Deans et al, 1992). Nevertheless, traditional morphologic criteria based upon evaluation by a pathologist are accurate for predicting recurrence in only $50 \%$ to $75 \%$ of the patients with nonmetastatic invasive colon carcinoma. Therefore, there is a need for additional, less subjective, independent factors to better predict outcome.

Cancer cells exhibit loss of genomic integrity. This may be, in part, related to chromosomal aberrations that can be secondary to disruption of cell cycle checkpoints (Cahill et al, 1998; Cheng and Loeb, 1993). Abnormal nuclear DNA content (aneuploidy), as determined by flow cytometry, reflects net changes in the number of chromosomes (Offerhaus et al, 1992;

Received August 31, 2000.

This work was supported by the Comision Interministerial de Ciencia y Tecnologia (Grants CICYT 98/42 and CICYT 00/81), the Fondo de Investigación Sanitaria (Grant FIS 94/37), and the Fundació La Marató de TV3 1994. RAR is a fellow of the Comissió Interdepartamental de Recerca i Innovació Tecnologica (CIRIT).

Address reprint requests to: Dr. Miguel A. Peinado, Institut de Recerca Oncologica, Hospital Duran i Reynals, Autovia Castelldefels $\mathrm{km} \mathrm{2,7,}$ 08907 L'Hospitalet, Barcelona, Spain.E-mail: mpeinado@iro.es
Remvikos et al, 1988) and has been proposed as a marker with prognostic applications. Nevertheless, no general agreement has been obtained regarding the routine clinical usefulness of aneuploidy in colorectal cancer (American Society of Clinical Oncology, 1996; Bauer et al, 1993). Studies in large series of samples indicate a lack of association between ploidy and survival (Tang et al, 1995; Zarbo et al, 1997). An explanation for the discrepancies in study results may be an inappropriate use of the DNA content of the most aneuploid cell population as the measurement of the aneuploidy of the tumor. This DNA index (DI) is based on experimental evidence suggesting that cells that are more aneuploid are likely to have higher malignant potential (Duesberg et al, 1998; Lengauer et al, 1997), resulting in increased tumor aggressiveness (Ciftone and Fidler, 1981). However, other studies have demonstrated that more invasive (metastatic) cells do not necessarily arise from the most aneuploid population (Scott et al, 1988; Tollenaar et al, 1997). Cells with a highly abnormal karyotype may be aberrant cells that are not necessarily viable (Scott et al, 1988). Tumors contain heterogeneous populations of cells and it is the aneuploidy heterogeneity, rather than the maximum degree of aneuploidy, that is associated with poorer outcome in different types of tumors (reviewed in Heppner and Miller, 1998). Thus, multiple sampling would be necessary to have an accurate estimation of the aneuploidy of the tumor (Bauer et al, 
1993). However, this is time consuming, expensive, and probably unfeasible for routine clinical application. The analysis of the S-phase fraction may be a more powerful prognostic parameter than DI (Bauer et al, 1993), but the inaccuracy of its calculation in aneuploid paraffin samples because of the overlapping of subpopulations limits its utility.

We developed a new index that, although not as accurate as multiple sampling, is an approximate measurement of the global aneuploidy of the tumor. Because the size of the aneuploid subpopulation can vary considerably from one tumor to another, the novel aneuploidy index (Al) takes into account not only the abnormal DNA content of the aneuploid subpopulation (the classical DI) but also its spread in the tumor. It is easily calculated as the DNA index computed proportionally to the area of diploid and aneuploid peaks. This measurement is made at the invasive edge of the tumor, a region that probably contains the highest burden of malignancy. Furthermore, the $\mathrm{Al}$ assessment can be performed in accessible paraffinembedded samples.

The accurate assessment of aneuploidy is relevant in light of recent studies (Duesberg et al, 1999; Li et al, 2000; Rasnick and Duesberg, 1999) suggesting that aneuploidy is the genetic mechanism underlying malignant transformation. Duesberg et al (1999) hypothesize that aneuploidy causes an autocatalytic karyotype evolution, which implies chromosomal instability and the resulting heterogeneity of cancer cells. In line with the work of Lengauer et al (1998), a reliable measurement of genetic instability, aneuploidy in this case, should consider not only the state, but also the rate of karyotype evolution. Because the $\mathrm{Al}$ integrates both concepts, it may more accurately reflect the malignant potential of a tumor. To test this hypothesis, we used flow cytometry to analyze the DNA content at the invasive edge of tumors in a series of 131 colorectal carcinomas. A corrected $\mathrm{Al}$, reflecting the average DNA content of the tumor specimen, was calculated and the association of $\mathrm{Al}$ with the clinical outcomes was investigated.

\section{Results}

\section{Ploidy Analysis}

Flow cytometry analysis of the 131 cases included in the study (see Material and Methods) provided three different indexes of aneuploidy: the classic DI; the ratio of aneuploid to diploid cells or cell index $(\mathrm{Cl})$; and the $\mathrm{Al}$, a composite measurement of the two previous parameters. A detailed definition of these indexes is given in the Material and Methods section. Thirty-nine tumors $(29.8 \%)$ were classified as diploid and 92 $(70.2 \%)$ as aneuploid. Among the aneuploid tumors, 19 cases (20.6\%) were near diploid ( $\mathrm{DI}=1.1$ to 1.2$), 1$ case was hypodiploid ( $\mathrm{DI}=0.79)$, and 5 cases were multiploid (1 contained a hypodiploid population). The two cases with hypodiploid populations were excluded. Therefore, 129 cases were available for statistical analysis. In multiploid cases (see Material and
Methods) the $\mathrm{Cl}$ and $\mathrm{Al}$ were calculated considering all aneuploid populations, and the highest DNA content was used for the DI. The mean values $( \pm \mathrm{SD}$ ) for the three indexes of the 90 tumors constituting the aneuploid group were: $\mathrm{DI}=1.57 \pm 0.31$ (range: 1.10 to 2.28 ), $\mathrm{Cl}=0.38 \pm 0.20$ (range: 0.02 to 0.81 ), and $\mathrm{Al}=$ $1.20 \pm 0.17$ (range: 1.00 to 1.84). Near-diploid tumors had approximate $\mathrm{Cl}$ values because of overlapping distributions of near-diploid and diploid cell populations. Therefore, these tumors were not included in the $\mathrm{Cl}$ analysis, but were included for the Al study because the $\mathrm{Cl}$ inaccuracy had little effect on Al calculation because of the low DI value. Linear regression analysis revealed no correlation between $\mathrm{DI}$ and $\mathrm{Cl}$ (data not shown), reinforcing the notion that these indexes provide complementary information. As expected, both $\mathrm{DI}$ and $\mathrm{Cl}$ correlated with Al. Proliferation parameters were determined in diploid tumors. The mean values $( \pm \mathrm{SD})$ were: S-phase fraction: $12.77 \pm$ 5.39 (range: 3.71 to 28.00 ) and proliferation index: $0.23 \pm 0.12$ (range: 0.05 to 0.55 ).

\section{Clinicopathologic Associations}

Ploidy status (diploid versus aneuploid) did not show any statistically significant differences with respect to clinicopathologic parameters. In aneuploid tumors, increased Al was associated with advanced Dukes' stage $(p=0.042)$, presence of metastasis $(p=0.020)$, and a nearly significant association was observed with left-sided tumors $(p=0.076)$. High $\mathrm{Cl}$ values were associated with metastasis $(p=0.023)$ and advanced Dukes' stage $(p=0.069)$. In contrast, DI did not show any statistically significant association.

\section{Survival Analysis}

Clinicopathologic variables and the three ploidy parameters were evaluated as prognostic factors with respect to overall and disease-free survival. In this analysis, only radical surgical resection (R0) tumors were considered $(n=108)$. Among clinicopathologic variables, only Dukes' stage was a predictor of poorer overall and disease-free survival (Table 1). The classification of the tumors based on the ploidy status (diploid versus aneuploid) did not show any association with survival (Table 1). This is the simplest tumor classification, which does not take into account the degree of aneuploidy. To test the working hypothesis (that the degree and extent of aneuploidy could affect tumor behavior), tumors were grouped into three categories: diploid, low aneuploid, and high aneuploid. Classifications were made according to the cutoff points for each index giving the highest prognostic efficiency after receiver operating characteristic (ROC) curve analysis. The cutoff values were Al greater than 1.14 (area under the curve: 0.675 , sensitivity: $80 \%$, specificity: 57\%), DI greater than 1.48 (area under the curve: 0.663 , sensitivity: $85 \%$, specificity: $50 \%$ ), and $\mathrm{Cl}$ greater than 0.17 (area under the curve: 0.529, sensitivity: 100\%, specificity: 18\%). The reported numbers correspond to the analysis of aneuploid 
Table 1. Univariate and Multivariate Overall Survival Analysis

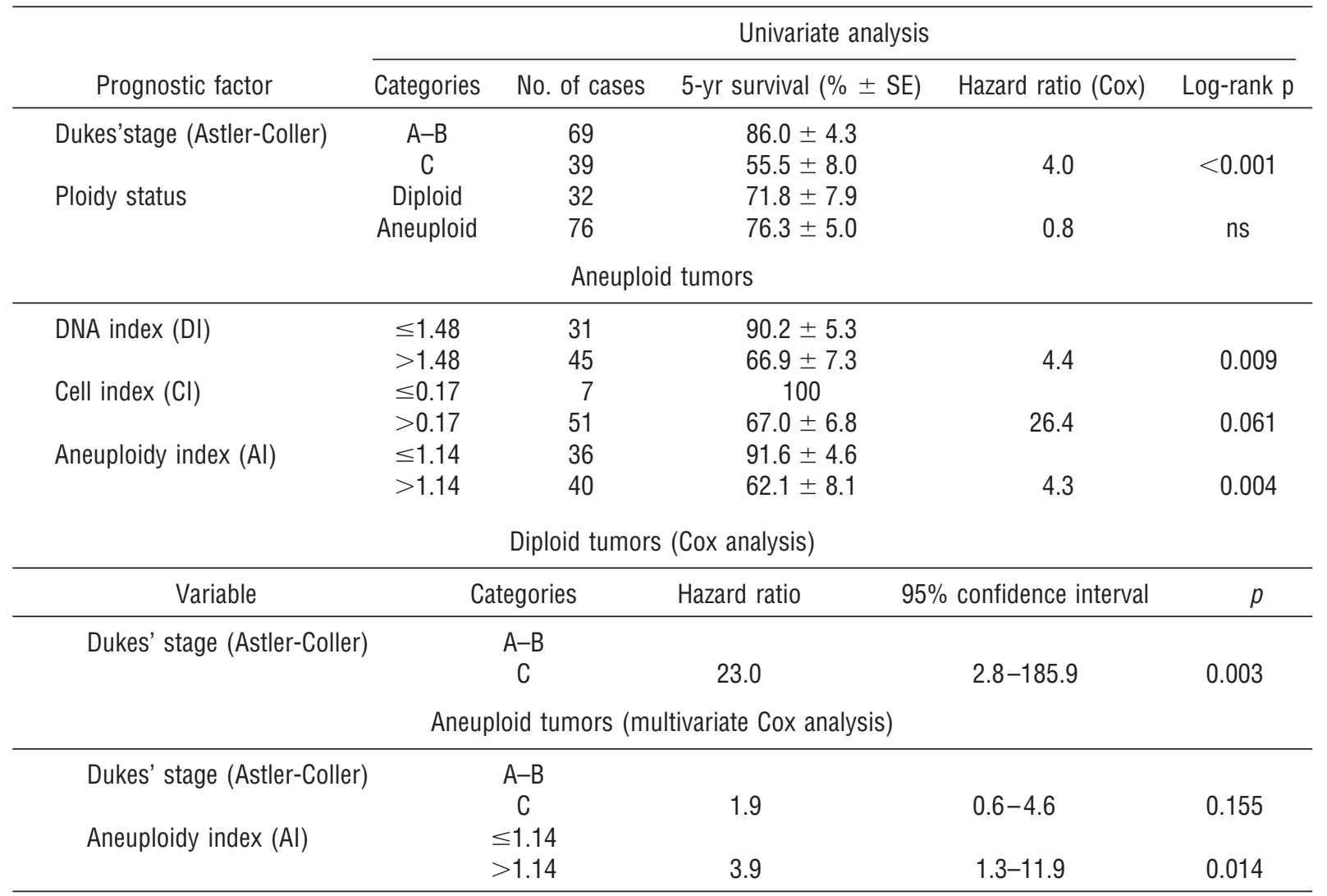

tumors, but identical cutoff values (with lower area under the curve values) were obtained if diploid cases were also included in the analysis.

The Kaplan-Meier curves for overall survival obtained with the three indexes were very similar, because the most significant classification was that based on the Al (Fig. 1a). Surprisingly, diploid tumors had an intermediate survival level, between low and high aneuploidy, which explains the lack of significance in the survival analysis of diploid versus aneuploid tumors. Sorting of diploid tumors according to Dukes' stage revealed a striking difference in survival (Fig. 1b and c). Interestingly, diploid tumors showed good prognosis in early Dukes' stages (Fig. 1b), whereas for Dukes' C stage survival decreased drastically, and was worse than for high Al tumors (Fig. 1c). Consequently, for further analyses, diploid tumors were classified as a separate group. This new classification based on both ploidy status and the Al allows a discrimination between tumors within the same stage but with different outcomes. The 5-year survival rate in patients classified by Dukes' stage (Table 1) is in agreement with previous data (Deans et al, 1992). However, for patients with tumors in Dukes' stages A and $\mathrm{B}$, a high $\mathrm{Al}$ identified a reduced survival rate $(68 \%$ versus 95\%) (Table 1 and Fig. 1). However, the survival rate $(80 \%)$ in patients with low $\mathrm{Al}$ and Dukes' grade $\mathrm{C}$ tumors is similar to the figures expected in less invasive stages (this work and Deans et al, 1992).

All aneuploidy parameters were indicators of a worse outcome in the univariate analysis (Table 1), except for the proliferation index (determined in diploid tumors, not shown). In the multivariate analysis, only $\mathrm{Al}$ was an independent prognostic factor (Table 1). These results indicate that the two primary factors analyzed, percentage of aneuploid cells and DNA content of the aneuploid population, both contribute to the determination of the outcome of the aneuploid tumors. However, only when both factors are combined in a single parameter, the Al, is clinically relevant information obtained. The multivariate analysis for diploid tumors indicates that Dukes' stage is a powerful prognostic predictor. However, the prognostic value of Dukes' stage is reduced and overcome by the Al for aneuploid tumors. The same conclusions were reached when disease-free survival was assessed.

Because adjuvant therapy may have influenced these observations, results were re-evaluated after classification for therapy. Although the setting of this study was not specifically designed to assess the impact of treatment on patient outcome, some interesting observations arose from our data. Only 4 (one with a high Al) of the 70 patients with Dukes' stage A and $B$ tumors received coadjuvant therapy (Table 2). All of the patients were alive at the end point of the follow-up. However in untreated patients with Dukes' stage $A$ and $B$ tumors, the prevalence of high $\mathrm{Al}$ values was increased in patients who died with disease (70\%) compared with living patients $(25 \%)(p=0.021)$ (Table 2). Univariate and multivariate survival analysis of this subset of untreated patients showed similar results to those reported for all RO cases (not shown). The 

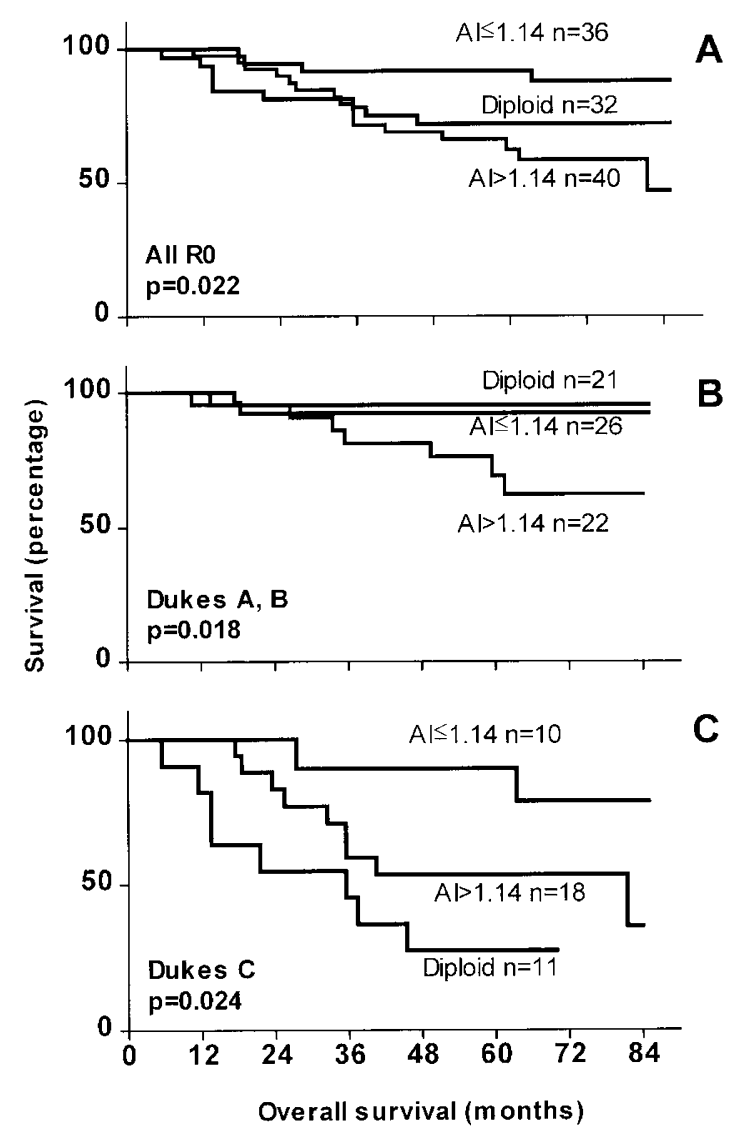

Figure 1.

Kaplan-Meier overall survival curves after classification for aneuploidy index (A). Cutoff point was set after receiver operating characteristic (ROC) curve analysis of all cases (>1.14). Curves have been traced with all patients (top), Dukes' stages A and B (center), and Dukes' stage C (bottom).

inclusion of $\mathrm{Al}$ in prognostic assessment would have reclassified 21 cases as high-risk, one-third of these patients (7 of 21 ; $33 \%$ ) died with disease; 44 patients (18 diploid and 26 low $\mathrm{Al}$ ) would have remained in the low-risk group, in which only 3 patients (7\%) died with disease. In patients with RO Dukes' grade C tumors, adjuvant therapy was given unless there was a contraindication, usually advanced age (mean age for treated patients: $61 \pm 14, n=18$; untreated patients: $71 \pm 13, n=22 ; p=0.025)$. Because of the low number of cases, it was not possible to draw conclusions. However, none of the treated patients with low Al tumors died with disease, and two of the six untreated patients died with disease. No differences were observed for diploid and high AI RO Dukes' grade C tumors.

To test the interaction between the two main parameters (Dukes' stage and Al) and survival, we analyzed the relationship of the smoothed hazard rate derived from a Cox model with these two variables (Fig. 2). The hazard rate increased with Al regardless of Dukes' stage. However, in Dukes' stages A and B tumors, increased Al clearly identified a subset of more aggressive tumors. The different behavior reflected in the curves for low- and high-Al tumors justifies the existence of a cutoff point at approximately $\mathrm{Al}=1.14$. In diploid tumors, Dukes' stage was a more resolute prognostic predictor than in aneuploid cases, and the hazard rate for diploid Dukes' grade $\mathrm{C}$ tumors was the highest of all groups. This analysis confirms the principal role of the $\mathrm{Al}$ in the outcome of aneuploid tumors, especially for Dukes' grade A and B tumors, and highlights the bad prognosis of diploid Dukes' grade C tumors.

\section{Discussion}

Genomic instability exists within tumors with genetic heterogeneity. Variant cells emerge throughout tumor evolution, and clones most suited to survive are selectively enriched. Heterogeneity within neoplasm is essential to the tumor's ability to survive, grow, and metastasize. This heterogeneity is probably responsible for the failure of otherwise effective therapeutic strategies (Heppner and Miller, 1998; Leith and Dexter, 1986; Vinyals et al, 1999). It is assumed that a correlation exists between increased genomic damage and malignant behavior (Arribas et al, 1997; Kern et al, 1989). Different molecular approaches have been used to quantify the extent of genomic disruption along the tumor and its association with identifiable clinical manifestations. Increased levels of chromosomal imbalances throughout the genome (Arribas et al, 1997; Kern et al, 1989; Offerhaus et al, 1992) and deregulation of gene expression (Tortola et al, 1999b) are associated with tumor aggressiveness in human colorectal cancer. However, the technical complexity of these approaches precludes their routine application. Flow cytometry analysis of aneuploidy, usually referred to as DI, seem to be a simpler alternative, but has proved of limited value (American Society of Clinical Oncology, 1996).

In this work, we have used the Al as a comprehensive estimate of the average chromosomal disruption

Table 2. Survival in Relation to the Coadjuvant Treatment Received and the Aneuploidy Index ${ }^{a}$

\begin{tabular}{|c|c|c|c|c|}
\hline \multirow[b]{2}{*}{ Status at follow-up end-point } & \multicolumn{2}{|c|}{ Treated } & \multicolumn{2}{|c|}{ Untreated $^{b}$} \\
\hline & Alive & Dead with disease & Alive & Dead with disease \\
\hline Diploid & 3 & 0 & 17 & 1 \\
\hline Low Al & 0 & 0 & 24 & 2 \\
\hline High Al & 1 & 0 & 14 & 7 \\
\hline
\end{tabular}

${ }^{a}$ Only patients with low-grade tumors (Dukes' stages A and B) and with no remnant disease (R0) were considered.

${ }^{b}$ One patient with a hypodiploid tumor (and dead with disease) was not included in the table. Chi-square test considering only untreated patients; $p=.021$. 


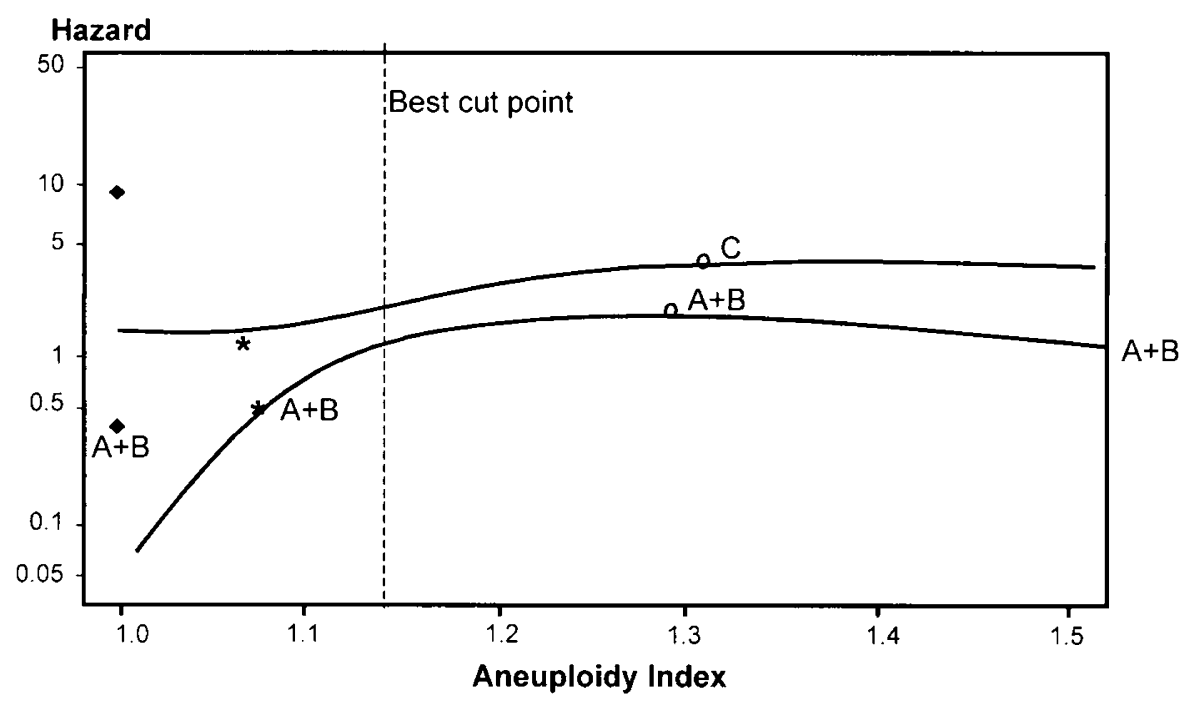

Figure 2.

Hazard curves for aneuploid tumors according to Dukes' stage. The vertical dashed line represents the cutoff point (>1.14) obtained in the ROC curve analysis for Al. Aneuploid tumors located to the left of this line are classified as low Al, and to the right, high Al. Hazards for diploid (rhombi), low Al (asterisks), and high Al (circles) tumors are indicated.

of the tumor cells. We hypothesized that a composite index of both the DNA content of the aneuploid peak and the proportion of aneuploid tumor cells would be a more reliable indicator of the intrinsic chromosomal instability than the conventional DI. To minimize the bias produced by contaminating normal cells in the Al calculation, ploidy analysis was performed on selected areas from the infiltration front. Necrotic tissue or areas with a high inflammatory component were discarded after exhaustive mapping, allowing the supposition that most of the cells contributing to the diploid peak were neoplastic. Despite tumor cell enrichment, all samples contained a small population of nontumor cells, which were detected in the histograms because of their distinct dispersion characteristics and were excluded from analysis. To confirm that the cells selected were of epithelial origin, a subset of cases with available optimal cryogenic temperature (OCT)-embedded material were analyzed using cytokeratin and DNA double immunoreactivity. Highly immunoreactive cells displayed diploid to aneuploid ratios similar to those obtained in the analyses described above (not shown), demonstrating the correctness of selection criteria. This experimental design and the high quality of the samples minimized the impact of technical limitations and therefore were critical in obtaining reliable results.

The Al has been shown to be an independent prognostic factor in colorectal tumors, being the only DNA content index to offer clinically useful information. The maximal differences were observed in Dukes' stage A and B tumors, where there is a need to identify those patients more likely to benefit from adjuvant therapy. Some interesting observations on the treatment impact on patient outcome arise from our data. The inclusion of the Al in prognostic assessment would have implied the reclassification of 21 cases as having a high-risk of recurrence. Although these results must be treated with caution because of the low number of cases and because the study was not designed for this purpose, our data suggest that this novel aneuploidy measurement might improve conventional pathologic classification.

The remarkable correlation between $\mathrm{Al}$ and poor prognosis probably reflects the unstable character of tumors with high Al values. In these tumors, it is likely that a greater number of tumor cells (represented by $\mathrm{Cl}$ ) are able to accumulate more genetic alterations (resulting in a higher DI), which increases the probability of variant and, occasionally, more aggressive clones within the tumor. Thus, it would be the cumulated genetic heterogeneity of the tumor that determines its malignant potential (Heppner and Miller, 1998). In contrast, tumors with a reduced number of aneuploid cells (low $\mathrm{Cl}$ ) with high $\mathrm{DI}$ values would have lower overall instability and, therefore, less potential for progression. Because only the degree of DNA content of the most aberrant cell population is taken into account in the DI, this limitation may underlie the lack of prognostic significance observed in this and other studies, as discussed above.

The observed correlation between increased chromosomal instability and malignant behavior (Arribas et al, 1997; Kern et al, 1989), also seen in the present study as assessed by $\mathrm{Al}$, might reflect two opposite situations: (i) that, in a given tumor, cells harboring the most abnormal karyotype are the most invasive and are selected for during distal dissemination (Duesberg et al, 1998; Lengauer et al, 1997); or (ii) that the presence of aneuploid clones is a symptom of the global chromosomal instability of the tumor, the more abnormal tumor cells are not necessarily viable (Scott et al, 1988). The aneuploidy profile of the primary tumor is often reproduced in its corresponding metastases, as found in unpublished observations (RA Risques and MA Peinado), and by Tollenaar et al (1997). 
Additionally, diploid tumor cells are present in most aneuploid primary tumors and metastases, as found here, in unpublished observations (RA Risques and MA Peinado), and by Bergstrom et al (1995). Therefore, it is likely that diploid cells have a critical role in tumor progression.

Consequently, estimations of tumor aggressiveness associated to genomic instability must consider the genetic damage of all tumor cell subpopulations. Lengauer et al (1998) introduced the concepts of "state" and "rate" to explain the significance of genomic instability on tumor development and progression. The presence of any aneuploid population in a tumor might be regarded as a "state", a manifestation of genomic instability not necessarily directly related to tumor progression. The aneuploid "rate" (the parameter determining the degree of instability) is better defined by the Al, because the Al measures the degree of abnormality (DNA content) taking into account the relative occurrence of abnormality (relative proportion of diploid to aneuploid cells). Therefore, a higher mutation rate (more chromosomal alterations per cell and an increased proportion of aneuploid cells) would result in an enhanced tumor heterogeneity and, eventually, an enhanced tumor progression (Lengauer et al, 1998). In the same sense, ploidy heterogeneity, as an outcome of chromosomal instability, has been associated with poor prognosis in colorectal cancers (Cosimelli et al, 1998; Flyger et al, 1999).

However, we observed different biologic behaviors among diploid tumors. Diploid tumors display specific genetic profiles (Dutrillaux, 1995; Fujiwara et al, 1998; Ionov et al, 1993; Offerhaus et al, 1992) and are likely to use different "progression engines" (Lengauer et al, 1998; Perucho et al, 1994) than aneuploid tumors (Li et al, 2000). Although diploid tumors have good prognoses in early stages, once distant metastases emerge, these tumors become highly malignant, suggesting the presence of an efficient mechanism of invasion and dissemination independent of aneuploidy. The previously reported association of diploid status with good prognosis, reviewed in Bauer et al (1993) and American Society of Clinical Oncology (1996), should be reexamined in light of our results. Furthermore, distinct molecular pathways are manifest in diploid tumors. One of these pathways includes tumors with ubiquitous microsatellite instability, which have a better prognosis (Gonzalez-Garcia et al, 2000; Halling et al, 1999; Lothe et al, 1993; Thibodeau et al, 1993). In our series, only seven patients (four diploid and three aneuploid) had microsatellite instability, six patients (all Dukes' stage A and B) had good outcomes and one (diploid, Dukes' stage C) died at six months of follow-up (not shown). Unfortunately, their low number precluded drawing further conclusions.

The aneuploidy progression pathway has been recently defined as an autocatalytic karyotype evolution that eventually generates preneoplastic and neoplastic cells (Duesberg et al, 1999; Li et al, 2000; Rasnick and Duesberg, 1999). Viability of abnormal karyotypes is likely to be lower than that of diploid cells. Therefore, the evolution is slow and clonal ( $\mathrm{Li}$ et al, 2000). A congruous inference from these observations could be that aneuploidy is the phenotype of the underlying chromosomal instability (regardless of a causative or consequential role of aneuploidy). This inference fits with our results. Therefore, our measurement of aneuploidy, Al, seems to be a sensible manifestation of genetic instability and is predictive of malignant behavior. Hazard smoothing in aneuploid tumors (Fig. 2) may be explained by a harmful effect of excessive levels of chromosomal instability on cell viability.

In summary, the novel aneuploidy index, $\mathrm{Al}$, is a comprehensive marker of tumor cell genomic instability, particularly of chromosome instability. Al seems to be a key parameter in predicting the malignant potential of colorectal tumors, and identifies a subgroup of patients that may benefit from more aggressive therapies. The correlation of $\mathrm{Al}$ with increased tumor aggressiveness is consistent with Foulds' and Nowell's postulates defining the generation of heterogeneity and cell selection as the underlying mechanisms of tumor progression (reviewed by Leith and Dexter, 1986).

\section{Material and Methods}

\section{Patients and Samples}

Between July 1991 and June 1994, 151 patients preoperatively diagnosed with colorectal cancer at the Hospital de la Santa Creu i Sant Pau, Barcelona, were prospectively included in a study designed to evaluate the prognostic value of specific genetic alterations and an estimation of overall genetic damage assessed by various techniques. Inclusion criteria were: a) electively resected primary adenocarcinomas; b) obtainment of fresh, paired normal mucosa and tumor samples within 2 hours of tumor removal; and c) no postoperative death. Inclusion in the study did not influence the adjuvant treatment given. The study protocol was approved by the Ethics Committee. No chemotherapy or radiotherapy was given before surgery in these patients (Tortola et al, 1999a). One hundred thirty-four of the 151 cases yielded high quality DNA (amenable for subchromosomal genomic damage assessment by DNA fingerprinting) from both paired normal and tumor samples (not shown). In these cases, flow cytometry analysis was attempted. In three tumors, analysis was not performed because of the poor quality of the available paraffin-embedded sections. Therefore, 131 cases were included in this study. Distributions for all clinicopathologic and genetic parameters considered were indistinguishable between excluded and included patients. The cases comprised 75 male and 56 female patients with a mean age of $66 \pm 12$ years (range: 33 to 96 years). Forty-one tumors were located in the right colon and 90 in the left colon including the rectum. The distribution of the carcinomas according to Dukes' classification was $18 \mathrm{~A}$ and $\mathrm{B} 1,55 \mathrm{~B} 2$ and B3, $41 \mathrm{C}$, and $17 \mathrm{D}$. At the last follow-up (January 1999), 72 patients were alive without disease, 7 dead without disease, 1 alive with disease and 51 had died because of disease. For 
survival analysis, only tumors from patients undergoing a R0, defined by the absence of macroscopic or microscopic remnant disease, not by Dukes' stage D, were considered ( $n=108$ tumors). Mean follow-up was $65 \pm 14$ months (range: 19 to 85 months).

\section{Flow Cytometry DNA Analysis}

DNA flow cytometry was performed from formalinfixed paraffin-embedded tissue blocks. Fifty micrometer sections were cut from microdissected regions of the tumor infiltration front (Fig. 3) with approximately $75 \%$ tumor cell content, as assessed by visual examination of hematoxylin and eosin stained preparations. Special care was taken to discard areas with necrotic tissue or harboring a high inflammatory component. DNA content was determined as previously described (Petriz et al, 1996) using an EPICS-XL-MCL flow cytometer (Coulter Electronics, Hialeah, Florida). List mode files were processed using the Mod Fit software package (Verity Software House, Inc., Topsham, Maine). Normal mucosa was used as an external control, showing a mean coefficient of variation (of the diploid G0/G1 peaks) of $3.43 \pm 0.39$. Despite tumor cell enrichment, all samples contained a number of nontumor cells. The distinct dispersion characteristics of the nontumor cells (ie, less side- and forwardscattering) allowed identification of the nontumor cells after histogram mapping. These nontumor cells were excluded from ploidy analysis. Moreover, this cell population provided an internal diploid control for every sample. Aneuploidy was defined as the presence of a nonapoptotic population of nuclei with any profile distinct from the diploid pattern $(\leq 0.9, \geq 1.1)$. DNA aneuploidy was expressed by three different indexes (Fig. 4): DI, the classical parameter to indicate aneuploidy, defined as the ratio of DNA content of the
G0/G1 peak of the tumor cell population to the diploid reference; $\mathrm{Cl}$, the measurement of the ratio between aneuploid cells and diploid cells (both in G0/G1 phases) regardless of the DNA content of the aneuploid peak (a tumor with $\mathrm{Cl}=1$ has the same proportion of diploid and aneuploid cells) (Fig. 4); and $\mathrm{Al}$, which integrates the two previous concepts, representing the average DNA content of the analyzed specimen (Fig. 4). An illustrative example of the flow cytometry analysis including index calculations is also depicted. Proliferation analysis was performed only for diploid tumors because of the unreliability of these measurements in the aneuploid histograms caused by overlapping populations. Two parameters were used: the S-phase fraction (\%S), which indicates the percentage of cells in $\mathrm{S}$ phase, and the proliferation index $(\mathrm{Pl})$, which is more accurate because it also includes cells in G2M. PI was calculated as: PI = (percent of S-phase cells + percent of G2M cells)/ G0G1 cells.

\section{Statistical Analysis}

Contingency tables were analyzed by Fisher's exact test or $\chi^{2}$ test. Statistical differences between means of quantitative variables were analyzed with unpaired $t$ tests or ANOVA, as appropriate. Disease-free and overall survival distributions were estimated by the Kaplan-Meier method and were compared using the log-rank test. Univariate and multivariate analyses were performed using the Cox proportional hazards model. Prognostic value of aneuploidy indexes was evaluated by considering them as quantitative variables and smoothing the relationship with restricted cubic splines. In this way, nonlinearity could be graphically assessed. Also, to summarize these results, cutoff values offering the maximum prognostic effi-
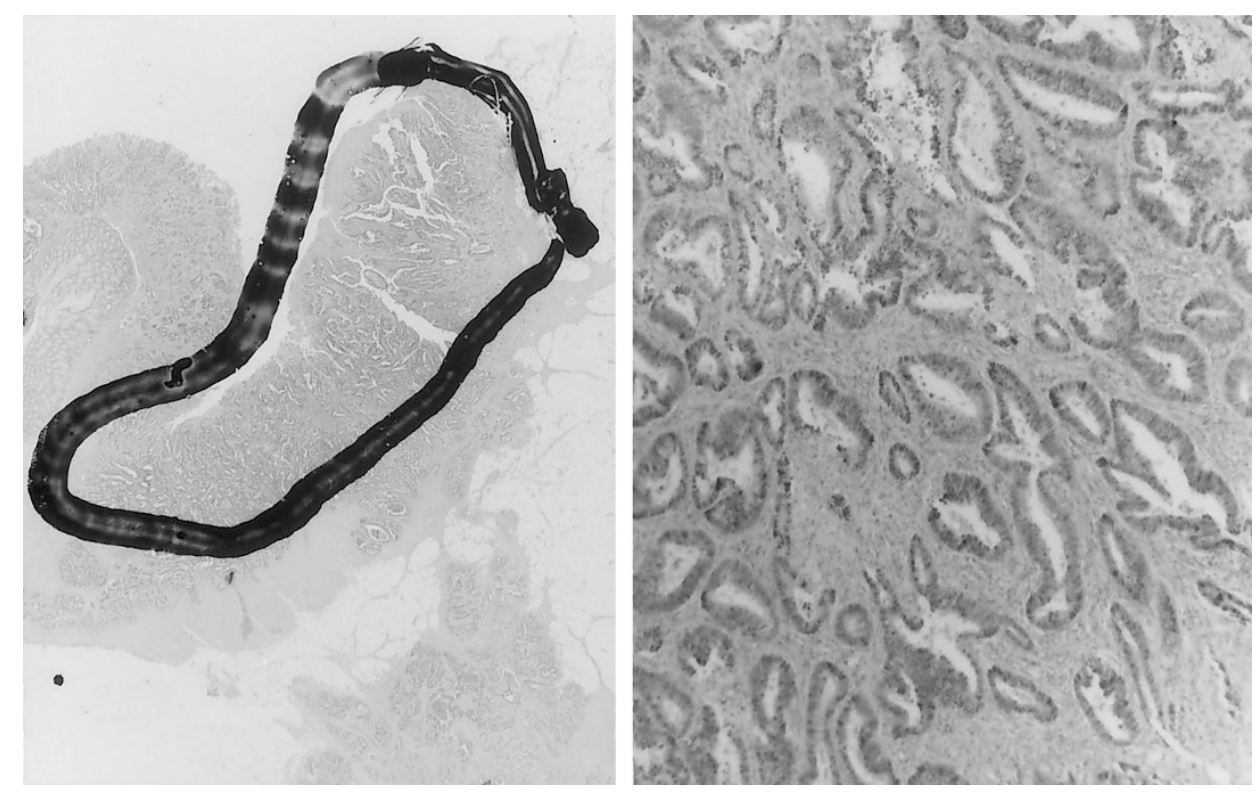

Figure 3.

(Left) Low-power magnification of an infiltrating carcinoma. The enclosed area corresponds to the invasive edge and was microdissected for flow cytometry analysis. (Right) An enlarged photomicrograph of the microdissected area is depicted. 


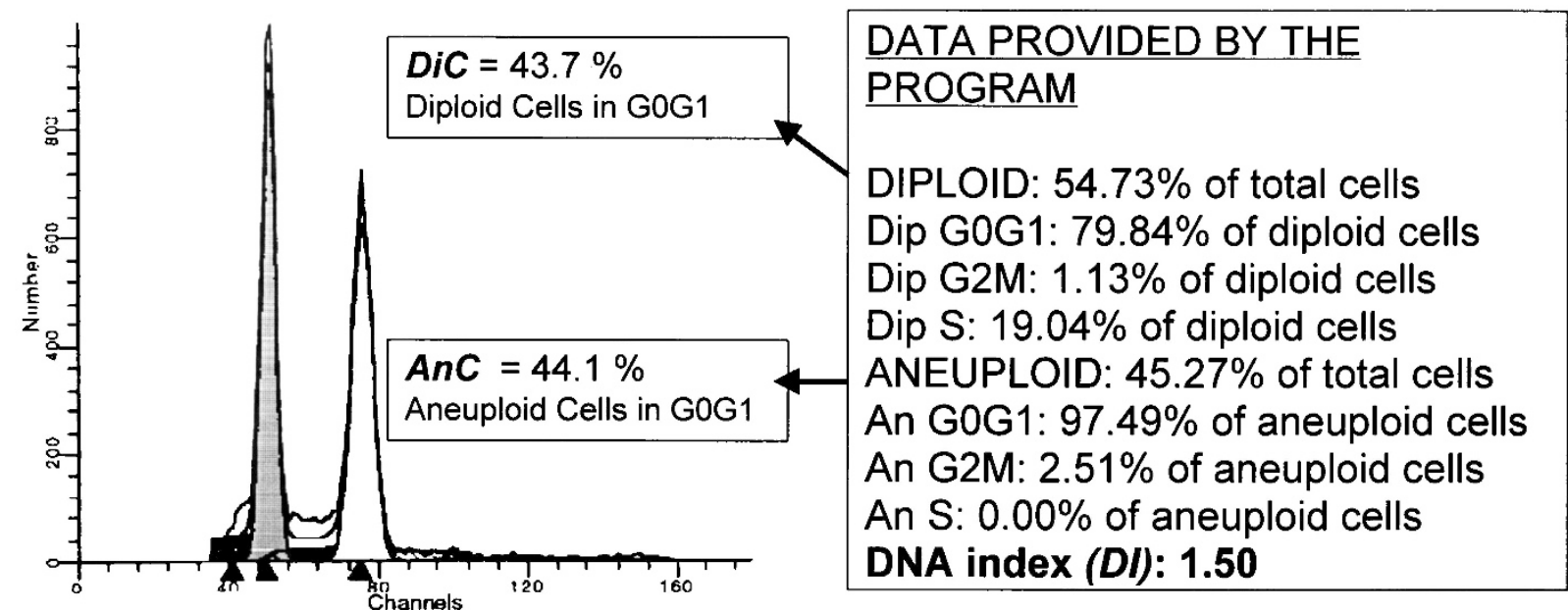

CALCULATION Cell Index $(\mathbf{C I})=\frac{A n C}{A n C+D i C}=\frac{44.1}{44.1+43.7}=\mathbf{0 . 5 0}$

Aneuploidy Index $(\mathrm{AI})=\frac{A n C \bullet D I+D i C \bullet 1}{A n C+D i C}=\frac{44.1 \bullet 1.5+43.7 \bullet 1}{44.1+43.7}=\mathbf{1 . 2 5}$

Figure 4.

Illustrative example of DNA content flow cytometry analysis in a colorectal tumor sample. Graph and primary parameters were generated using the Mod Fit program (see "Materials and Methods"). Three different aneuploidy indexes (DI, Cl, and Al) were calculated according to the formulas depicted.

ciency were calculated for $\mathrm{DI}, \mathrm{Cl}, \mathrm{Al}, \% \mathrm{~S}$, and PI after ROC curve analysis of disease-free and overall survival. All reported $p$ values were derived from twosided statistical tests.

\section{Acknowledgements}

We thank Maria Julià, Agnès Figueras, Felip Vilardell, and Gemma Aiza for excellent technical support.

\section{References}

American Society of Clinical Oncology (1996). Clinical practice guidelines for the use of tumor markers in breast and colorectal cancer. J Clin Oncol 14:2843-2877.

Arribas R, Capella G, Tortola S, Masramon L, Grizzle WE, Perucho M, and Peinado MA (1997). Assessment of genomic damage in colorectal cancer by DNA fingerprinting: Prognostic applications. J Clin Oncol 15:3230-3240.

Bauer KD, Bagwell B, Giaretti W, Melamed M, Zarbo RJ, Witzig TE, and Rabinovitch PS (1993). Consensus review of the clinical utility of DNA flow cytometry in colorectal cancer. Cytometry 14:486-491.

Bergstrom C, Emdin S, Roos G, and Stenling R (1995). DNA content in colorectal carcinoma: A flow cytometric study of the epithelial fraction. Anal Cell Pathol 8:287-295.

Cahill DP, Lengauer C, Yu J, Riggins GJ, Willson JK, Markowitz SD, Kinzler KW, and Vogelstein B (1998). Mutations of mitotic checkpoint genes in human cancers. Nature 392:300-303.

Cheng KC and Loeb LA (1993). Genomic instability and tumor progression: Mechanistic considerations. Adv Cancer Res 60:121-156.
Ciftone MA and Fidler IJ (1981). Increasing metastatic potential is associated with increasing genetic instability of clones isolated from murine neoplasms. Proc Natl Acad Sci USA 78:6949-6952.

Cosimelli M, D’Agnano I, Tedesco M, D’Angelo C, Botti C, Giannarelli D, Vasselli S, Cavaliere F, Zupi G, and Cavaliere R (1998). The role of multiploidy as unfavorable prognostic variable in colorectal cancer. Anticancer Res 18:1957-1965

Crissman JD, Zarbo RJ, Ma CK, and Visscher DW (1989). Histopathologic parameters and DNA analysis in colorectal adenocarcinomas. Pathol Annu 24:103-147.

Deans GT, Parks TG, Rowlands BJ, and Spence RA (1992). Prognostic factors in colorectal cancer. Br J Surg 79:608613.

Duesberg P, Rasnick D, Li R, Winters L, Rausch C, and Hehlmann R (1999). How aneuploidy may cause cancer and genetic instability. Anticancer Res 19:4887-4906.

Duesberg P, Rausch C, Rasnick D, and Hehlmann R (1998). Genetic instability of cancer cells is proportional to their degree of aneuploidy. Proc Natl Acad Sci USA 95:1369213697.

Dutrillaux B (1995). Pathways of chromosome alteration in human epithelial cancers. Adv Cancer Res 67:59-82.

Flyger HL, Larsen JK, Nielsen HJ, and Christensen IJ (1999). DNA ploidy in colorectal cancer, heterogeneity within and between tumors and relation to survival. Cytometry 15:38: 293-300.

Fujiwara T, Stolker JM, Watanabe T, Rashid A, Longo P, Eshleman JR, Booker S, Lynch HT, Jass JR, Green JS, Kim H, Jen J, Vogelstein B, and Hamilton SR (1998). Accumulated clonal genetic alterations in familial and sporadic colorectal carcinomas with widespread instability in microsatellite sequences. Am J Pathol 153:1063-1078. 
Gonzalez-Garcia I, Moreno V, Navarro M, Martí-Ragué J, Marcuello E, Benasco C, Campos O, Capella G, and Peinado MA (2000). Standardized approach for microsatellite instability detection in colorectal carcinomas. J Natl Cancer Inst 92:544-549.

Halling KC, French AJ, McDonnell SK, Burgart LJ, Schaid DJ, Peterson BJ, Moon-Tasson L, Mahoney MR, Sargent DJ, O'Connell MJ, Witzig TE, Farr GH, Goldberg RM, and Thibodeau SN (1999). Microsatellite instability and $8 p$ allelic imbalance in stage B2 and C colorectal cancers. J Natl Cancer Inst 91:1295-1303.

Heppner GH and Miller FR (1998). The cellular basis of tumor progression. Int Rev Cytol 177:1-56.

Ionov Y, Peinado MA, Malkhosyan S, Shibata D, and Perucho M (1993). Ubiquitous somatic mutations in simple repeated sequences reveal a new mechanism for colonic carcinogenesis. Nature 363:558-561.

Kern SE, Fearon ER, Tersmette KWF, Enterline JP, Leppert M, Nakamura $Y$, White R, Vogelstein B, and Hamilton SR (1989). Clinical and pathological associations with allelic loss in colorectal carcinoma [published correction appears in JAMA 1989, 262:1952]. JAMA 261:3099-3103.

Leith JT and Dexter DL (1986). Mammalian tumor cell heterogeneity. Boca Raton: CRC Press Inc., 97-135.

Lengauer C, Kinzler KW, and Vogelstein B (1997). Genetic instability in colorectal cancers. Nature 386:623-627.

Lengauer C, Kinzler KW, and Vogelstein B (1998). Genetic instabilities in human cancers. Nature 396:643-649.

Li R, Sonik A, Stindl R, Rasnick D, and Duesberg P (2000). Aneuploidy vs gene mutation hypothesis of cancer: Recent study claims mutation but is found to support aneuploidy. Proc Natl Acad Sci USA 97:3236-3241.

Lothe RA, Peltomaki P, Meling Gl, Aaltonen LA, NystromLahti M, Pylkkanen L, Heimdal K, Andersen TI, Moller P, Rognum TO, Fossa SD, Haldorsen T, Langmark F, Brogger A, de la Chapelle A, and Borrensen AL (1993). Genomic instability in colorectal cancer: Relationship to clinicopathological variables and family history. Cancer Res 53:5849-5852.

Offerhaus GJA, De Feyter EP, Cornelisse CJ, Tersmette KWF, Floyd J, Kern SE, Vogelstein B, and Hamilton SR (1992). The relationship of DNA aneuploidy to molecular genetic alterations in colorectal carcinoma. Gastroenterology 102:1612-1619.

Perucho M, Peinado MA, lonov Y, Casares S, Malkhosyan S, and Stanbridge E (1994). Defects in replication fidelity of simple repeated sequences reveal a new mutator mechanism for oncogenesis. Cold Spring Harb Symp Quant Biol 59:339 348.
Petriz J, Tugues D, and Garcia-Lopez J (1996). Relevance of forward scatter and side scatter in aneuploidy detection by flow cytometry. Anal Cell Pathol 10:243-252.

Rasnick D and Duesberg PH (1999). How aneuploidy affects metabolic control and causes cancer. Biochem J 340:621630.

Remvikos Y, Muleris M, Vielh P, Salmon RJ, and Dutrillaux B (1988). DNA content and genetic evolution of human colorectal adenocarcinoma. A study by flow cytometry and cytogenetic analysis. Int J Cancer 42:539-543.

Scott CA, Desinan L, Avellini C, Bardus P, Rimondi G, Rizzi V, and Beltrami CA (1988). DNA index shift with disease progression in colorectal adenocarcinoma: A morphological and flow cytometry study. Hum Pathol 29:482-490.

Tang R, Ho YS, You YT, Hsu KC, Chen JS, Changchien CR, and Wang JY (1995). Prognostic evaluation of DNA flow cytometry and histopathologic parameters of colorectal cancer. Cancer 76:1724-1730.

Thibodeau SN, Bren G, and Schaid D (1993). Microsatellite instability in cancer of the proximal colon. Science 260:816819.

Tollenaar RAEM, Bonsing BA, Kuipers-Dijkshoorn NJ, Hermans J, van de Velde CJH, Cornelisse CJ, and Fleuren GJ (1997). Evidence of clonal divergence in colorectal carcinoma. Cancer 79:1304-1314.

Tortola S, Marcuello E, Gonzalez I, Reyes G, Arribas R, Aiza G, Sancho FJ, Peinado MA, and Capella G (1999a). p53 and $\mathrm{K}$-ras gene mutations correlate with tumor aggressiveness but are not of routine prognostic value in colorectal cancer. J Clin Oncol 17:1375-1381.

Tortola S, Marcuello E, Risques RA, Gonzalez S, Aiza G, Capella G, and Peinado MA (1999b). Overall deregulation in gene expression as a novel indicator of tumor aggressiveness in colorectal cancer. Oncogene 30:4383-4387.

Vinyals A, Peinado MA, Gonzalez-Garrigues M, Monzo M, Bonfil RD, and Fabra A (1999). Failure of wild-type p53 gene therapy in human cancer cells expressing a mutant p53 protein. Gene Ther 6:22-33.

Zarbo RJ, Nakhleh RE, Brown RD, Kubus JJ, Ma CK, and Mackowiak P (1997). Prognostic significance of DNA ploidy and proliferation in 309 colorectal carcinomas as determined by two-color multiparametric DNA flow cytometry. Cancer 79:2073-2086. 